\title{
Neurobrucellosis Presenting with Features of Demyelinating Disorder in a Pediatric Patient
}

\author{
Muhammad Saeed ${ }^{1}$, Ahmed Al Bishri ${ }^{1}$, Sanaa Gammaa ${ }^{1}$, Abdul Qadir $^{1}$, Saleh Al-Qahtani ${ }^{2}$ \\ and Ayed Abdullah Shati ${ }^{2}$ \\ ${ }^{1}$ Department of Pediatric Medicine, Armed Forces Hospital, Southern Region, Saudi Arabia \\ ${ }^{2}$ King Khalid University, Abha Southern Region, Saudi Arabia
}

\begin{abstract}
Brucellosis is an endemic disease in Saudi Arabia, which can present with variable clinical manifestations. It is a zoonotic disease transmitted from animals to humans. Brucellosis is a multisystemic disease that can present with any system involvement; and neurobrucellosis is a serious complication, sometimes leading to permanent neurological deficit, if treatment is not started promptly. Herein, we present a 6-year boy with neurobrucellosis, who developed demyelination of cerebral white matter and presented with fever and seizures.
\end{abstract}

Key Words: Neurobrucellosis, Demyelination, White matter.

How to cite this article: Saeed M, Bishri AA, Gammaa S, Qadir A, Qahtani SA, Shati AA. Neurobrucellosis Presenting with Features of Demyelinating Disorder in a Pediatric Patient. J Coll Physicians Surg Pak 2022; 32(02):247-249.

\section{INTRODUCTION}

Brucellosis is considered as the most common zoonotic infectious disease across the world. It is endemic in many regions of the world, including south Asia, the Middle-East, and the Arabian peninsula. ${ }^{1}$ However, the incidence of brucellosis is decreasing rapidly in Saudi Arabia due to tremendous development of healthcare facilities, immunisation of sheep and cattle, and the general improvement of health standards.

Brucellosis is a multi-systemic infectious disease that can present with a variety of clinical signs and symptoms. In the United States, 100 to 200 cases of human brucellosis are reported annually. ${ }^{2}$ Neurobrucellosis is a most serious complication of brucellosis, reported in $3-5 \%$ of patients in literature. ${ }^{3}$ Neurobrucellosis can present as meningoencephalitis, polyneuritis, radiculitis, or myelitis. Acute neurobrucellosis is usually due to direct invasion of the organisms in the central nervous system; while in subacute or chronic form of the disease, bacteria may remain in the cells and result in late clinical manifestations. ${ }^{4}$

Neurobrucellosis is rarely associated with diffuse demyelination in adult patients. However, this finding has not been reported very commonly in children.

Correspondence to: Dr. Muhammad Saeed, Department of Pediatric Medicine, Armed Forces Hospital, Southern

Region, Saudi Arabia

E-mail: muhammad964@hotmail.com

Received: December 16, 2019; Revised: April 21, 2021;

Accepted: April 26, 2021

DOI: https://doi.org/10.29271/jcpsp.2022.02.247
There are lot of disparities in the diagnostic criteria of neurobrucellosis, and only case reports and short series have been motioned in the literature. ${ }^{5}$ Herein, we present a 6 -yearboy with neurobrucellosis, who developed demyelination of cerebral white matter and presented with fever and seizures.

\section{CASE REPORT}

A 6-year boy was admitted in our hospital with history of fluctuating fever for the last six months, associated with mild headache and anorexia. On the day of admission, he had frequent episodes of vomiting with two episodes of generalised tonic clonic convulsions of short duration. There was no history of altered sensorium, irritability or any weakness. There was also no history of raw milk consumption but he belonged to rural area and was associated with livestock rearing. After admission, temperature was documented to be up to $102^{\circ} \mathrm{F}$.

On examination, the child was well oriented and there were no signs of meningeal irritation and focal neurological deficits. His all accessible cranial nerves were intact; and examination of gait was also normal. Rest of the systemic examination was also normal. Blood chemistry results showed normal glucose level, normal renal and liver functions.

Cerebrospinal fluid (CSF) analysis revealed protein of 1129 $\mathrm{mg} / \mathrm{dl}$ and glucose $35 \mathrm{mg} / \mathrm{dl}$ (concomitant blood glucose: 106 $\mathrm{mg} / \mathrm{dl}$ ). CSF microscopic examination showed total white blood cell (WBC) count of $36 / \mathrm{cm}^{3}$ with $30 \%$ neutrophils and $70 \%$ lymphocytes. CSF culture was negative and no organism was identified on gram staining. Viral panel in CSF was also unremarkable with negative oligoclonal bands. Brucella titer upon admission was: Brucella Abortus 1:10240 (normal value less 
than 1:80), and Brucella Melitensis 1:5120 (normal value less than $1: 80$ ). His blood culture was negative.

Magnetic resonance imaging (MRI) of brain without contrast showed bilateral symmetrical confluent areas of altered signal intensity of the cerebral subcortical and deep white matter on T2-weighted and FLAIR sequences and there was no gadolinium enhancement (Figure 1). MRI spine was reported normal.
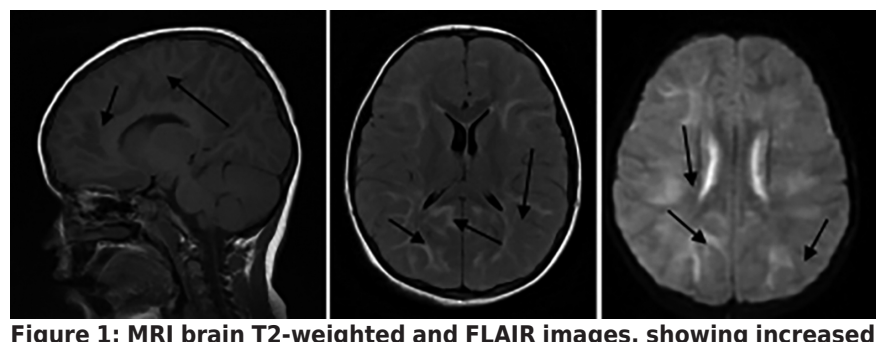

Figure 1: MRI brain T2-weighted and FLAIR images, showing increased signals intensity in the cerebral white matter.

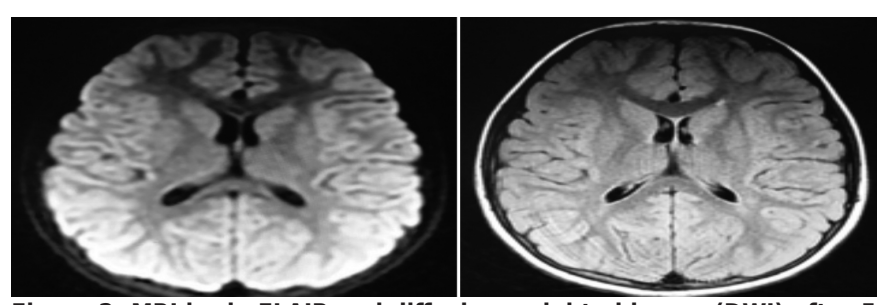

Figure 2: MRI brain FLAIR and diffusion-weighted image (DWI) after 5 months, showing normal white matter.

Based on history, clinical examination, and investigations, a diagnosis of demyelinating type of neurobrucellosis was made, and the patient was started on combination of co-trimoxazole and rifampicin. One dose of intravenous immunoglobulin (IVIG), $2 \mathrm{gm} / \mathrm{kg}$ over two days, was also given. Treatment was completed for six months and follow-up brucella titer was normal. Follow-up MRI after five months showed complete resolution of white matter lesions bilaterally (Figure 2).

\section{DISCUSSION}

Brucellosis is a common infectious disease in the Arabian Peninsula and Mediterranean countries. Endemic areas for brucellosis include countries of the Mediterranean basin, Middle-East, Central Asia, China, the Indian subcontinent, sub-Saharan Africa, and parts of Mexico and Central and South America. ${ }^{2,6}$ Neurobrucellosis is a serious complication of systemic brucellosis. In pediatric systemic brucellosis, CNS involvement is not very common, and only $0.8 \%$ of the children are affected. The diagnosis of neurobrucellosis needs a high index of suspicion in endemic areas, especially in children, where clinical symptoms are not very specific.

However, history of exposure to live stock and consumption of raw milkis also very important to supportthe diagnosis with radiological findings. Here, we report a rare type of neurobrucellosis with bilateral cerebral white matter demyelination, which was diagnosed based on clinical features, brucella titer and CNS radiological findings.

There are five different types of neurobrucellosis including meningoencephalitis, meningovascular involvement, CNS demyelination, peripheral neuropathy, and increased intracranial pressure. CNS involvement in neurobrucellosis may also mimic other inflammatory conditions, such as acute disseminated encephalomyelitis (ADEM), CNS vasculitis and multiple sclerosis(MS).

In a study on patients with neurobrucellosis, criteria for diagnosis were defined as: 1) neurobrucellosis clinical manifestations; 2) CSF abnormality (lymphocytosis, decreased glucose, and increased protein); 3) positive anti-brucella antibody in CSF or serum; 4) clinical response to empirical therapy; and 5) no other diagnosis compatible with signs and symptoms. ${ }^{5}$

So according to literature, our patient had definite neurobrucellosis, based on clinical features, laboratory investigations and radiological findings.

Demylination is the rarest form of neurobrucellosis. The clinical symptoms of neurobrucellosis vary and are reported as non-pathognomonic insomestudies. ${ }^{4}$ Fever is the mostcommon clinical symptom of neuobrucellosis, and might be associated with headache, vomiting and seizures, as we have observed in our patient. In the past, it was described that changes in white matter, noticed in neurobrucellosis, were a result of demyelination, an observation that was confirmed by ensuing histopathological studies. Similarly, white matter changes have also been noticed in other patients from Saudi Arabia. ${ }^{7}$ In our patient, we also noticed bilateral cerebral demyelination of subcortical white matter sparing the corpus callosum.

Although the incidence of neurobrucellosis is not very frequent, but physicians should keep their mind open to identify it clinically. Early diagnosis and management not only reduces the morbidity, but also decreases the neurological sequalae.

Neurobrucellosis is a serious complication of brucellosis, and if not diagnosed early, it may lead to serious consequences. There should be a high index of suspicion in endemic areas. Early management can lead to a good prognosis with minor or no neurological sequalae.

\section{PATIENT'S CONSENT:}

Verbal consent was taken from the parents for publication of this report.

\section{CONFLICT OF INTEREST:}

The authors declared no conflict of interest.

\section{AUTHORS' CONTRIBUTION:}

All coauthors helped in searching related articles, analyzing, collecting information and writing discussion of the case report.

\section{REFERENCES}

1. Pappas G, Akritidis N, Bosilkovski M, Tsianos E. "Medical progress Brucellosis." Engl J Med 2005; 22:2325-67.

2. Buzgan T, Karahocagil MK, Irmak H, Irfan Baran A, Karsen $\mathrm{H}$, Evirgen $\mathrm{O}$, et al. Clinical manifestations and complications in 1028 cases of brucellosis: A retrospective evaluation and review of the literature. Int J Infect Dis 
2010; 14(6):469-78. doi: 10.1016/j.ijid.2009.06.031.

3. Dean AS, Crump L, Greter H, Hattendorf J, Schelling E, Zinsstag J. Clinical manifestations of human brucellosis: A systematic review and meta-analysis. PLoS Negl Trop Dis 2012; 6(12):1929. doi: 10.1371/journal.pntd.0001929.

4. Young EJ. Brucella species. In: Mandel I GL, Bennett JE, Dolin R, eds. Principles and practice of infectious diseases. 7th ed. Philadelphia: Churchill Livingstone, 2010:2921-5.

5. Asadipooya K, Dehghanian A, Omrani GH, Abbasi F. Short- course treatment in neurobrucellosis: A study in Iran. Neurol India 2011; 59(1):101-3. doi: 10.4103/0028-3886. 76879.

6. Seleem MN, Boyle SM, Sriranganathan N. Brucellosis: A reemerging zoonosis. Vet Microbiol 2010; 140(3-4):392-8. doi: 10.1016/j.vetmic.2009.06.021.

7. Al-Sous MW, Bohlega S, Al-Kawi MZ, Alwatban J, McLean DR. Neurobrucellosis: Clinical and neuroimaging correlation. AJNR Am J Neuroradiol 2004; 25(3):395-401. 